\title{
Trombólisis intravenosa exitosa en infarto cerebral agudo en paciente con historia de macroadenoma y apoplejía hipofisaria
}

\author{
Successful intravenous thrombolysis in acute cerebral infarction in a \\ patient with a history of macroadenoma and hypophysial stroke
}

\author{
Camilo Romero, ${ }^{\star}$ Mariana Gaviria, ${ }^{\ddagger}$ Jorge Andrés Mutis* \\ ${ }^{*} \mathrm{MD}$, Neurólogo. \\ ₹ MD, Residente de Neurología. \\ Universidad del Rosario, Fundación Cardioinfantil. Bogotá, Colombia.
}

\section{RESUMEN}

El tratamiento con activador tisular del plasminógeno intravenoso es probablemente recomendado para pacientes con infarto cerebral agudo que tienen una neoplasia intracraneal extraaxial, pero en aquéllos con historia de hemorragia intracraneal es potencialmente nocivo. No ha sido reportada en la literatura la administración de trombólisis intravenosa durante un infarto cerebral agudo en pacientes con historia de macroadenoma de hipófisis con apoplejía pituitaria y sangrado intratumoral. Presentamos el caso de una paciente joven con un evento isquémico cerebral agudo en el territorio de la arteria cerebral media izquierda, porción M3, con puntaje de 14 de la National Institutes of Health Stroke Scale y 0 en la escala de Rankin modificada al ingreso, con historia de macroadenoma hipofisiario con apoplejía pituitaria y escaso sangrado intratumoral que exitosamente fue llevada a trombólisis intravenosa. La toma de decisiones sobre la administración intravenosa del activador tisular del plasminógeno para el manejo del infarto cerebral agudo debe estar basada en la evaluación de los potenciales riesgos y beneficios esperados caso por caso, teniendo en cuenta como marco de referencia las guías escritas sobre el tema.

Palabras clave: Infarto cerebral isquémico, macroadenoma hipofisiario, apoplejía pituitaria, trombólisis intravenosa, activador tisular del plasminógeno.

\section{ABSTRACT}

Thrombolysis with tissue plasminogen activator is probably recommended for patients with acute ischemic stroke and extra-axial intracranial neoplasm, but in those with a history of intracranial hemorrhage it is potentially harmful. Intravenous thrombolysis in acute ischemic stroke in patients with a history of pituitary macroadenoma and apoplexy with intratumoral bleeding has not been reported in the literature. We report the case of a young patient with an acute ischemic stroke in the territory of the left middle cerebral artery, M3 portion, National Institutes of Health Stroke Scale of 14 and modified Rankin scale of 0 at admission, with a history of pituitary macroadenoma and pituitary apoplexy and mild intratumoral bleeding successfully brought to intravenous thrombolysis. The decision to thrombolysis with tissue plasminogen activator for the management of acute ischemic stroke should be based on the evaluation of the expected risks and benefits on a case-by-case basis taking into account the current guidelines.

Keywords: Ischemic stroke, pituitary macroadenoma, pituitary apoplexy, intravenous thrombolysis, tissue plasminogen activator.

Abreviaturas: $\mathrm{ACM}=$ Arteria cerebral media, $\mathrm{ASA} / \mathrm{AHA}=$ American Heart Association/American Stroke Association, ASPECT = Alberta Stroke Program Early CT Score, ERm = Escala modificada de Rankin, NIHSS = National Institutes of Health Stroke Scale, RMN = Resonancia magnética nuclear, TPA = Activador tisular del plasminógeno. 


\section{INTRODUCCIÓN}

La aprobación del uso de trombólisis intravenosa con TPA, en 1996, significó uno de los más grandes avances en el manejo del infarto cerebral agudo, sin embargo, no todos los pacientes son candidatos para su administración. ${ }^{1}$ En los últimos 25 años las contraindicaciones se han modificado para ampliar su uso y las guías de la American Heart Association/American Stroke Association (ASA/AHA) del 2019 recomiendan discutir los riesgos potenciales contra los beneficios esperados durante el proceso de decisión de la trombólisis intravenosa. ${ }^{2}$ En las guías se describe que el tratamiento con TPA intravenoso se recomienda probablemente para pacientes con infarto cerebral agudo que tienen una neoplasia intracraneal extraaxial, pero en aquéllos con historia de hemorragia intracraneal es potencialmente nocivo. ${ }^{2}$ Series de casos han evaluado los desenlaces y la seguridad de la administración intravenosa de TPA en pacientes con antecedente de sangrado intracraneal. ${ }^{3}$ Sin embargo, la administración de trombólisis intravenosa con TPA en infarto cerebral agudo en pacientes con historia de macrodenoma de hipófisis con apoplejía pituitaria y sangrado intratumoral no ha sido reportada en la literatura.

El infarto cerebral en pacientes con tumores hipofisarios puede ocurrir por un vasoespasmo o compresión de estructuras vasculares adyacentes; en este escenario el tratamiento del infarto cerebral debe ir dirigido a la lesión neoplásica. ${ }^{4}$ Cuando el infarto cerebral no se explica por los mecanismos anteriores, el uso de trombólisis intravenosa con TPA podría mejorar el pronóstico de estos pacientes. A continuación, presentamos un caso de un infarto cerebral agudo en paciente con antecedente de macroadenoma de hipófisis con apoplejía pituitaria y escaso sangrado intratumoral, que fue tratada exitosamente con la administración intravenosa de TPA.

\section{REPORTE DE CASO}

Presentamos el caso de una mujer de 34 años que ingresa por cuadro clínico de instauración súbita de 2 horas de evolución con afasia no fluida y hemiparesia derecha. Como antecedente médico tiene historia de macroadenoma hipofisiario con apoplejía pituitaria y escaso sangrado intratumoral 11 meses previos a su ingreso (Figura 1).

EI NIHSS de ingreso fue de 14 puntos, la tomografía de cráneo simple inicial (Figura 2) mostraba un ASPECT 10/10, y una lesión neoplásica hipofisaria $5 \times 6 \mathrm{~mm}$ sin sangrado intratumoral. La angiotomografía de vasos intracraneales evidenciaba una oclusión distal a la bifurcación de la arteria cerebral media (ACM) izquierda porción M3 (Figura 3). La glucometría de ingreso y sus signos vitales estaban dentro de límites normales.

Se analizaron las neuroimágenes previas (hospitalización por apoplejía pituitaria) y las actuales, en conjunto con el neurólogo intervencionista de la institución y se descartó la posibilidad de procedimiento endovascular. Se consideró a la paciente como candidata a manejo con TPA a dosis de $0.9 \mathrm{mg} / \mathrm{kg}$. Ésta no presentó deterioro neurológico durante la administración de TPA intravenoso ni posterior a ella. La tomografía de cráneo simple efectuada 24 horas postrombólisis no mostró transformación hemorrágica ni sangrado intratumoral.

Durante la estancia hospitalaria de 17 días la paciente recupera parcialmente su expresión verbal y fuerza; mantiene un puntaje en la NIHSS de 7 y en la escala modificada de Rankin de 2 al egreso. En la imagen de resonancia magnética nuclear cerebral durante la hospitalización no se documentó crecimiento tumoral ni evidencia de sangrado intratumoral posterior a la trombólisis (Figura 4). En los estudios de estratificación se documenta que la causa
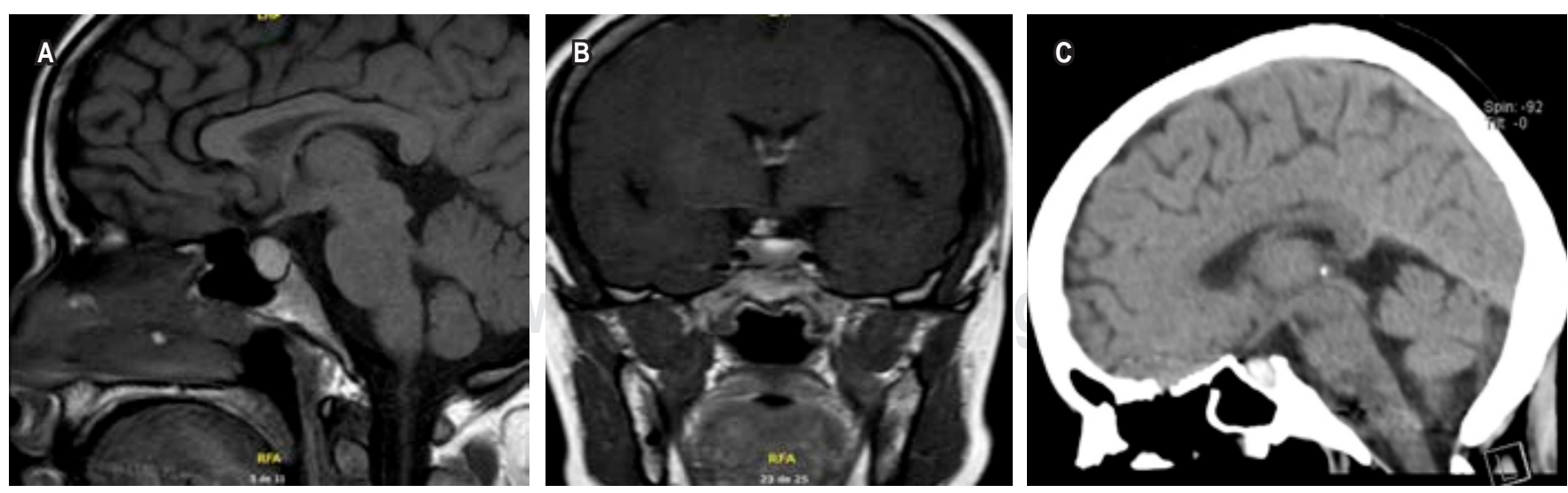

Figura 1: A) Resonancia magnética nuclear $(\mathrm{RMN})$ cerebral, corte sagital, T1 simple: aumento de tamaño en la glándula hipófisis (15 × $9 \times 13$ mm), con área hiperintensa que sugiere sangrado. B) RMN silla turca, corte coronal, T1 con contraste: realce al medio de contraste. C) Tomografía de cráneo simple, corte sagital: aumento de tamaño de glándula hipofisiaria, con aumento en la densidad que sugiere sangrado. 

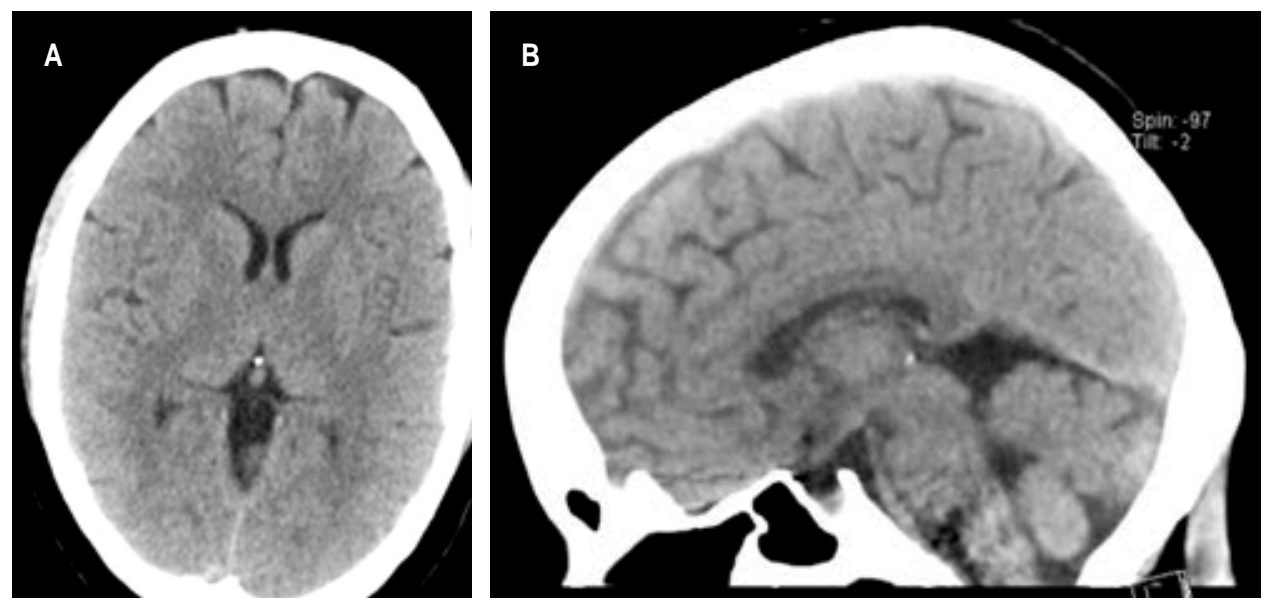

Figura 2:

A) Tomografía de cráneo simple, corte axial: ASPECT 10/ 10. B) Tomografía de cráneo simple, corte sagital, sin evidencia de sangrado en hipófisis.

del infarto es indeterminada. Se egresa a la paciente con prevención secundaria con monoantiagregación con ácido acetil salicílico y atorvastatina.

\section{DISCUSIÓN}

Reportamos el caso de una paciente joven con un evento isquémico cerebral agudo en el territorio de la ACM izquierda porción M3, NIHSS de 14 y ERm de 0 al ingreso, con historia de macroadenoma hipofisiario con apoplejía pituitaria y escaso sangrado intratumoral exitosamente tratada con trombólisis intravenosa con TPA.

Hay datos clínicos que se oponen a la administración intravenosa de TPA para el manejo del infarto cerebral agudo de nuestra paciente. Las guías de la ASA/AHA contraindican la administración del TPA en pacientes con historia de sangrado intracraneal. ${ }^{2}$ Adicionalmente un macroadenoma tiene un riesgo de apoplejía hipofisiaria del $8-10 \%$ que puede implicar sangrado intratumoral. ${ }^{5}$ Los factores predisponentes a la apoplejía pituitaria incluyen pruebas de estimulación endocrina, tratamiento con bromocriptina/cabergolina, trauma craneal, embarazo, radioterapia y, tal vez, alteraciones en la coagulación. ${ }^{6,7}$ Ahmed y Semple han descrito la isquemia cerebral como una potencial complicación de la apoplejía pituitaria por oclusión compresiva de la arteria carótida interna en el seno cavernoso o vasoespasmo. ${ }^{8}$ La administración de TPA intravenoso no tendría utilidad en estos casos. Por último, no hay evidencia de la seguridad del uso de TPA en pacientes con esta patología. Rubinshtein y colaboradores describen un caso de trombólisis intravenosa con TPA en una paciente de 66 años con infarto agudo de miocardio. ${ }^{9}$

Las guías de la ASA/AHA describen que el tratamiento con TPA intravenoso probablemente se recomienda para pacientes con infarto cerebral agudo que tienen una neo- plasia intracraneal extraaxial. ${ }^{2}$ La apoplejía pituitaria con sangrado intratumoral es un fenómeno vascular que al parecer se deriva del crecimiento tumoral con una relativa insuficiencia de irrigación o por compresión/distorsión de la vasculatura que suple la glándula..$^{6,7}$ El sangrado resultante ocurre por necrosis isquémica hemorrágica. ${ }^{5-7}$ En nuestra paciente, la apoplejía pituitaria con escaso sangrado intratumoral había sucedido hace 11 meses, el tamaño del tumor se había reducido y no tenía historia de alteraciones en la coagulación ni historia clínica de apoplejía pituitaria. Las neuroimágenes actuales descartaron compresión o vasoespasmo de la arteria carótida interna y no evidenciaron sangrado intratumoral. Por último, nuestra paciente estaba dentro de la ventana terapéutica para la administración de trombolítico intravenoso, no

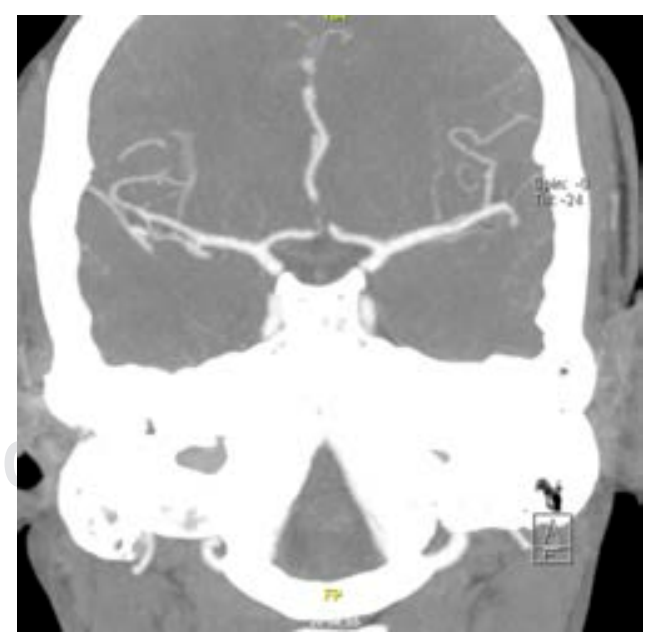

Figura 3: Tomografía de vasos arteriales intracraneal, corte sagital, obstrucción en flujo arterial en la porción distal de M3 izquierda, sin signos de compresión o vasoespasmo de la arteria carótida interna izquierda. 


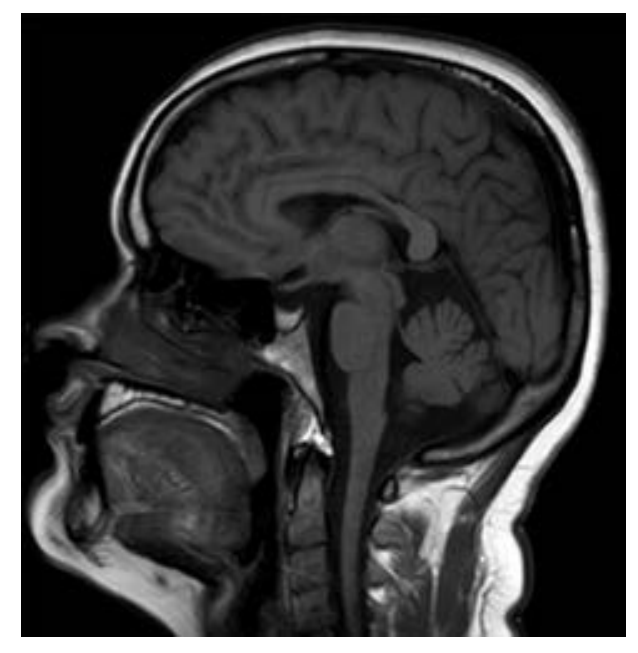

Figura 4: Resonancia magnética nuclear (RMN) cerebral, corte sagital $\mathrm{T} 1$, sin evidencia de crecimiento tumoral.

presentaba contraindicaciones adicionales a las expuestas y no era una candidata ideal para la realización de un procedimiento endovascular para el manejo de su evento isquémico cerebral agudo.

Teniendo en cuenta lo anterior, se decidió administrar trombólisis intravenosa con TPA a dosis de $0.9 \mathrm{mg} / \mathrm{kg}$ para el manejo del evento isquémico agudo de la ACM izquierda porción M3. La intervención se consideró exitosa por la mejoría de 7 puntos en la NIHSS, un puntaje de 2 en la ERm y ausencia de sangrado intratumoral o apoplejía pituitaria al egreso. Éste es el primer caso de trombólisis intravenosa con TPA para un evento isquémico cerebral agudo en paciente con historia de macroadenoma hipofisiario con apoplejía pituitaria y escaso sangrado intratumoral, reportado en la literatura.

\section{CONCLUSIÓN}

Durante el proceso de la toma de decisiones sobre la administración de TPA intravenosa para el manejo del infarto cerebral agudo recomendamos valorar los potenciales riesgos y beneficios esperados caso por caso teniendo en cuenta como marco de referencia las guías escritas sobre el tema. La decisión debe tener como fundamento el probable beneficio más que los predictores de desenlace.

\section{REFERENCIAS}

1. Brainin M, Wolf-Dieter H. Textbook of Stroke Medicine. United Kingdom: Cambridge University Press; 2010.

2. Powers WJ, Rabinstein AA, Ackerson T, Adeoye OM, Bambakidis NC, Becker K, et al. Guidelines for the early management of patients with acute ischemic stroke:2019 update to the 2018 Guidelines for the Early Management of Acute Ischemic Stroke: A Guideline for Healthcare Professionals from the American Heart Association/ American Stroke Association. Stroke 2019;50:e344-e418.

3. Lee SH, Kim BJ, Han MK, Park TH, Lee KB, Lee BC, et al. Should we exclude acute stroke patients with previous intracerebral hemorrhage from receiving intravenous thrombolysis? Int J Stroke 2016;11(7):783-790.

4. Serramito R, Santín JM, Román P, Pita L, González L. Infarto cerebral tras apoplejía pituitaria: descripción de un caso y revisión de la literatura. Neurocirugía 2016;27:310-314.

5. Vargas G, González B, Guinto G, Mendoza V, López B, Zepeda $\mathrm{E}$, et al. Pituitary apoplexy in nonfunctioning pituitary macroadenomas: a case-control study. Endocr Prac 2014;20(12):1274-1280.

6. Nawar RN, Abdel-Mannan D, Selman WR, Arafah BM. Pituitary tumor apoplexy: a review. J Intensive Care Med 2008;23(2):75-90.

7. Kaplan B, Day AL, Quisling R, Ballinger W. Hemorrhage into pituitary adenomas. Surg Neurol 1983;20(4):280-287.

8. Ahmed SK, Semple PL. Cerebral ischemia in pituitary apoplexy. Acta Neurochir (Wien) 2008;150(11):1193-1196.

9. Rubinshtein R, Jaffe R, Flugelman MY, Karkabi B, Lewis BS. Thrombolysis in patients with a brain tumor. Heart 2004;90(12):1476. 